\title{
Orientation Imaging Microscopy: Sample Preparation for Heavily Drawn Copper wires
}

\author{
D.R. Waryoba and P.N. Kalu \\ FAMU-FSU College of Engineering, and \\ National High Magnetic Field Laboratory, Tallahassee, FL 32310.
}

The use of Orientation Imaging Microscopy (OIM) as a microcharacterization technique has evolved into a standard analytical tool for studying deformed or processed materials $[1,2]$. OIM technique relies on accurate orientation data via electron backscattered pattern (EBSP) measurements to recreate the microstructure of materials. In spite of the considerable advances that have been made on this technique, it is still inadequate for use in the study of heavily deformed materials [3]. This is partly due to lack of appropriate specimen preparation methods, and partly due to the interference of the fine substructures or/and grain size with the formation of the EBSPs in such materials [3]. It is therefore important to assess the extent to which specimen preparation can improve the quality of OIM data obtained from heavily deformed materials.

The OFHC Cu used in this study was drawn at room temperature to a true strain of 3.2, and the OIM measurements were carried out in an environmental scanning electron microscope (ESEM), model E3 with OIM capability. Fig. 1a presents a typical EBSP, which was generated from specimen prepared by conventional metallographic method (method 1). The method involved a sequence of mechanical polishing of the specimen: a) grinding on 400 grit and 1200 grit $\mathrm{SiC}$ papers, b) polishing with a $3 \mu \mathrm{m}$ diamond paste and c) polishing on a $0.25 \mu \mathrm{m}$ OP-S suspension. It is evident that the EBSP did not show any discernible diffraction pattern, which made automatic indexing impossible. Hence, it is not surprising that the corresponding OIM grain orientation map of Fig. 1b is unreliable. The presence of several white spots in the orientation map was an indication of large number of bad points in the data.

A second specimen preparation method (method 2) used consisted of a sequence of mechanical polishing followed by chemical etching. The etching was carried out in a solution containing an equal part of ammonium hydroxide $\left(\mathrm{NH}_{4} \mathrm{OH}\right)$ and hydrogen peroxide $3 \%\left(\mathrm{H}_{2} \mathrm{O}_{2}\right)$, via swabbing for about 10 - 15 seconds [4]. This procedure did not produce suitable specimen surface for OIM analysis, as can be seen in the EBSP and OIM orientation map of Figures $2 \mathrm{a}$ and $2 \mathrm{~b}$, respectively. The lack of diffraction pattern in the EBSP of Figure 2a clearly indicates that the sample was not adequately prepared for OIM analysis. Hence the Kikuchi bands could not be reliably indexed, and consequently, the corresponding OIM grain orientation map of Figure $2 \mathrm{~b}$ is not dependable. Electropolishing of mechanically polished samples did not produce distinguishable diffraction pattern either.

The inability to use conventional specimen preparation to produce good quality samples for OIM analysis led to the development of the new specimen preparation technique (method 3). The threestage method involves: a) mechanical polishing, b) chemical etching, and c) polishing on the vibratory machine for 2 hour. A typical diffraction pattern from the specimen polished by this technique is presented in Figure 3a. It is evident that the pattern was very clear and the Kikuchi bands were distinguishable and indexable. The resulting OIM grain orientation map shown in 
Figure $3 \mathrm{~b}$ was an excellent representation of the microstructure of the material. Quite noticeable is the distinct elongated fibrous grains, characteristic of wire drawn microstructure. It is reasonable to conclude that the specimen preparation technique developed was reliable for the OIM study of heavily deformed $\mathrm{OFHC} \mathrm{Cu}$.

\section{References}

[1] D. J. Dingley, Scanning electron microscopy, 1984, 569-575.

[2] J. A. Venables, and C. J. Harland, Philos. Mag. 27 (1972), 1193-1200.

[3] S. I. Wright and U. F. Kocks, ICOTOM-11 (eds. Z. Liang, L. Zuo and Y. Chu), 1996, 53-62.

[4] V. Voort, in Metallography: Principles and Practice" Mater. Sci. Eng. series, McGraw-Hill, 1984, pp. 622.

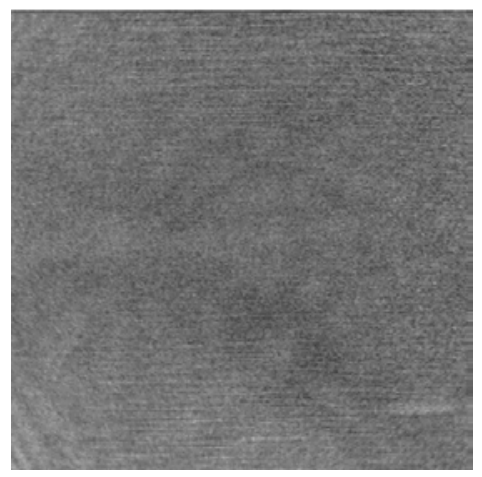

1(a)

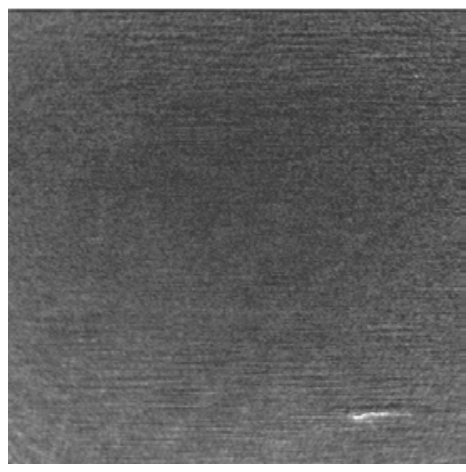

2(a)

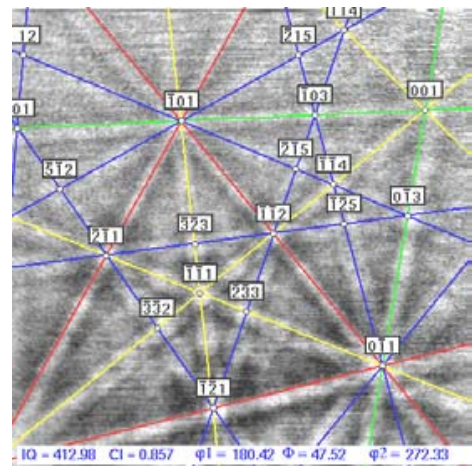

3(a)

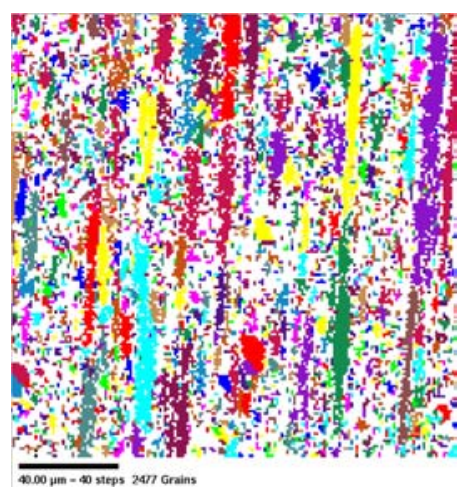

1(b)
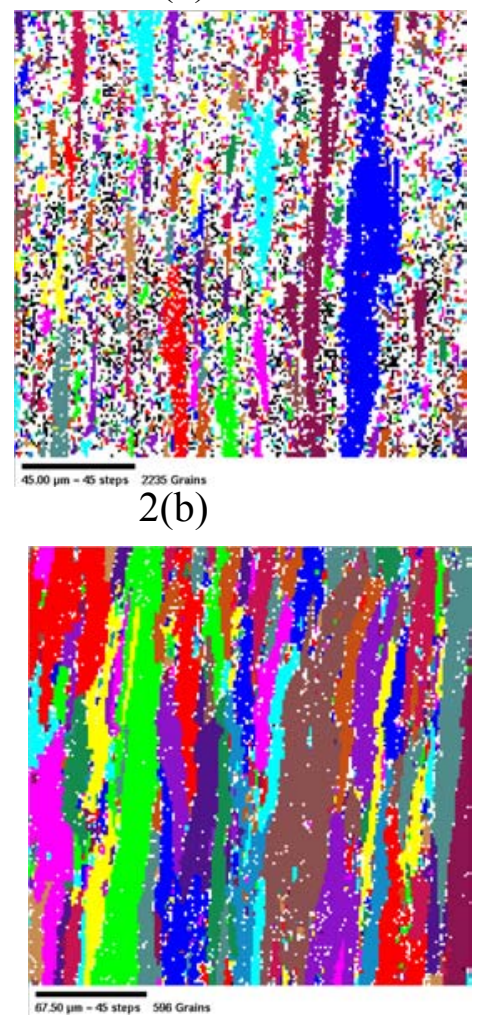

3(b)
Figure 1 a) OIM diffraction pattern, and b) corresponding grain orientation map generated from a sample prepared by method 1

Figure 2 a) OIM diffraction pattern, and b) corresponding grain orientation map generated from a sample prepared by method 2

Figure 3 a) OIM diffraction pattern, and b) corresponding grain orientation map generated from a sample prepared by method 3 